\title{
Studying the neural bases of prism adaptation using fMRI: A technical and design challenge
}

\author{
Janet H. Bultitude ${ }^{1,2,3}$ - Alessandro Farnè ${ }^{1,4}$ - Romeo Salemme ${ }^{1}$ - Danielle Ibarrola ${ }^{5}$. \\ Christian Urquizar $^{1}$ • Jacinta O'Shea ${ }^{3}$ • Jacques Luauté ${ }^{1,4}$
}

Published online: 30 December 2016

(C) The Author(s) 2016. This article is published with open access at Springerlink.com

\begin{abstract}
Prism adaptation induces rapid recalibration of visuomotor coordination. The neural mechanisms of prism adaptation have come under scrutiny since the observations that the technique can alleviate hemispatial neglect following stroke, and can alter spatial cognition in healthy controls. Relative to non-imaging behavioral studies, fMRI investigations of prism adaptation face several challenges arising from the confined physical environment of the scanner and the supine position of the participants. Any researcher who wishes to administer prism adaptation in an fMRI environment must adjust their procedures enough to enable the experiment to be performed, but not so much that the behavioral task departs too much from true prism adaptation. Furthermore, the
\end{abstract}

Jacinta O'Shea and Jacques Luauté contributed equally to this work.

Electronic supplementary material The online version of this article (doi:10.3758/s13428-016-0840-z) contains supplementary material, which is available to authorized users.

Janet H. Bultitude

j.bultitude@bath.ac.uk

1 ImpAct Team, Lyon Neuroscience Research Centre, INSERM U1028, CNRS UMR5292, University Claude Bernard Lyon I, Lyon, France

2 Department of Psychology, University of Bath, Claverton Down, Bath BA2 7AY, UK

3 Oxford Centre for Functional Magnetic Resonance Imaging of the Brain (FMRIB), Nuffield Department of Clinical Neurosciences, University of Oxford, Headley Way, Headington, Oxford OX3 9DU, UK

4 Hospices Civils de Lyon, Hôpital Henry Gabrielle, Mouvement et Handicap, Neuro-immersion, F-69000 Lyon, France

5 Centre d'Exploration et de Recherche Médicale par Emission de Positons, Imagerie du vivant, 69677 Bron cedex, France specific temporal dynamics of behavioral components of prism adaptation present additional challenges for measuring their neural correlates. We developed a system for measuring the key features of prism adaptation behavior within an fMRI environment. To validate our configuration, we present behavioral (pointing) and head movement data from 11 righthemisphere lesioned patients and 17 older controls who underwent sham and real prism adaptation in an MRI scanner. Most participants could adapt to prismatic displacement with minimal head movements, and the procedure was well tolerated. We propose recommendations for fMRI studies of prism adaptation based on the design-specific constraints and our results.

Keywords Prism adaptation · Hemispatial neglect · Functional imaging $\cdot \mathrm{fMRI}$

\section{Introduction}

Prism adaptation has been used for over a hundred years to investigate the coordination of sensory inputs and motor performance (Harris, 1965; Hay, Langdon, \& Pick, 1971; Hay \& Pick, 1966; Held \& Freedman, 1963; Held \& Mikaelian, 1964; von Helmholtz, 1962; Stratton, 1896; for a review see Redding \& Wallace, 1997). However, the precise neuronal circuitry that mediates this form of sensorimotor plasticity remains unclear. Some practical reasons for this are the difficulties inherent in capturing this dynamic form of visuomotor learning within the neuroimaging environment. A typical prism adaptation protocol begins with a person seated at a table with their hands resting close to their chest and two or more targets positioned at arm's length from their body. The participant is then fitted with goggles that contain prismatic lenses that displace the visual image laterally. The participant 
is asked to point to the targets one at a time, returning their hand to their chest between each pointing movement. If online correction is prevented through the use of rapid pointing movements and by occluding the early part of the movement, participants will at first miss the target, pointing erroneously in the direction of prismatic displacement, rather than to the true target location. With repeated pointing movements, however, these errors will reduce gradually, as participants adapt to the prismatic shift and correct their errors in the direction opposite to the optical shift. If the prisms are then removed and the participant is asked to point to a target without visual feedback of their pointing arm, their movement will now err in the direction opposite to the prismatic shift: the so-called adaptation "after-effect". This after-effect is the key behavioral change that is considered evidence that true adaptation (not merely strategic compensatory strategies) has occurred (Redding, Rossetti, \& Wallace, 2005; Redding \& Wallace, 1997).

To elaborate on the distinction between "true" prism adaptation and strategic compensatory strategies, prism adaptation involves several complex and interacting processes [see Redding \& Wallace (1997) for a comprehensive treatment of this topic]. Broadly speaking, these can be broken down into two main processes: a fast and somewhat deliberate re-aiming known as strategic recalibration, and a slower adjustment of low-level sensorimotor relationships known as realignment. These behavioral components can be studied by observing the evolution of two types of pointing errors over time: closed-loop and open-loop. During prism exposure, participants perform closed-loop pointing (CLP) in which they can see the target, their hand, and the terminal error. Thus CLP errors give a measure of the rate of error correction while prism adaptation is taking place. However, the evolution of the adaptation after-effect is measured through periodic openloop pointing (OLP) trials in which vision of the entire arm movement including the terminal pointing error is occluded from the participant's view. The trial-to-trial reduction in closed-loop pointing errors occurs through a blend of both strategic recalibration and sensorimotor realignment. In contrast, the magnitude of the OLP errors predominantly reflects sensorimotor realignment, especially if the contribution of strategic recalibration is minimized by using targets for OLP trials that are in a different location to the CLP targets.

The neural mechanisms of prism adaptation have come under particular scrutiny since Rossetti and his colleagues (1998) reported that adaptation to rightward-shifting prisms, which results in a leftward after-effect, can ameliorate the symptoms of left hemispatial neglect ("neglect"). Neglect is characterized by difficulty responding and orienting to information on the left side of the body and space (Heilman, Valenstein, \& Watson, 2000; Parton, Malhotra, \& Husain, 2004). Although the mechanism underlying the therapeutic effect of prism adaptation on neglect remains to be fully elucidated, sensorimotor realignment is thought to drive the neglect reduction since, for example, patients who show no adaptation after-effects also show no improvement (Frassinetti, Angeli, Meneghello, Avanzi, \& Ladavas, 2002). Patients with left spatial neglect following right hemisphere lesions show no changes in symptoms following adaptation to leftward-shifting prisms and also no adaptation after-effects (Luauté et al., 2012), suggesting that sensorimotor realignment is a necessary precursor to changes in spatial cognition. Understanding the changes in neural activity that occur in the brains of stroke patients during prism adaptation may provide insights into how this low-level sensorimotor adaptation can modulate high-level cognitive disorders. However, neglect improvements do not correlate with after-effects in terms of duration (Frassinetti et al., 2002) or magnitude (Sarri et al., 2008; Serino, Angeli, Frassinetti, \& Làdavas, 2006), and there is some evidence that the ability to correct pointing errors during prism exposure may better predict therapeutic outcome (Serino, Bonifazi, Pierfederici, \& Làdavas, 2007). Therefore, it would prove useful to disentangle the neural processes that underlie behaviors that take place during prism exposure (CLP trials, or error correction) from those that reflect sensorimotor realignment (OLP trials, or after-effect). Such efforts could also be informed through research in healthy controls, in whom prism adaptation has been shown to produce changes in spatial cognition that resemble some of those that are shown by neglect patients (Bultitude \& Woods, 2010; Loftus, Nicholls, Mattingley, \& Bradshaw, 2008; Loftus, Vijayakumar, \& Nicholls, 2009; Michel et al., 2003; Michel, Pisella, Prablanc, Rode, \& Rossetti, 2007; Nicholls, Kamer, \& Loftus, 2008). Mirroring the directionally-selective effects of prism adaptation in neglect patients, changes in spatial cognition in healthy controls almost exclusively follow adaptation to leftward-shifting prisms, although there have been isolated reports of such changes following adaptation to rightwardshifting prisms, too (Berberovic \& Mattingley, 2003; Striemer, Sablatnig, \& Danckert, 2006).

Although there have been a large number of behavioral studies investigating the effects of prism adaptation on spatial cognition in neurologically healthy participants and brainlesioned patients (for reviews, see Luauté, Halligan, Rode, Jacquin-Courtois, \& Boisson, 2006; Michel et al., 2003; Newport \& Schenk, 2012; Striemer \& Danckert, 2010), only a few groups have succeeded in using functional magnetic resonance imaging (fMRI) to study changes in brain activity induced by prism adaptation (Chapman et al., 2010; Danckert, Ferber, \& Goodale, 2008; Küper et al., 2014; Luauté et al., 2009). fMRI investigations of prism adaptation face several major challenges, which fall into two categories. First, challenges arise due to the need to accommodate the essential procedures of a prism adaptation task within the confines of the scanner environment. Second, the specific temporal dynamics of the behavioral components of prism adaptation - 
particularly the slow and cumulative sensorimotor realignment - present specific challenges for capturing their neural correlates.

With regard to physical challenges of accommodating prism adaptation in an fMRI environment, the confined space within the bore of the MRI scanner limits the participant's range of movement and hinders easy fitting and removal of prism goggles. Also, the supine position of the participant makes it difficult to provide direct vision of their pointing hand and the targets. Manual pointing also threatens the integrity of functional data by the possible introduction of head and arm movement artifacts (Culham et al., 2003; Grootoonk et al., 2000; Liao, McKeown, \& Krolik, 2006). Finally, the presence of a strong magnetic field precludes the use of ferromagnetic material and magnetic motion-tracking devices. Any researcher who wishes to administer prism adaptation in an fMRI environment must therefore adjust their procedures enough to allow the experiment to be done, but not so much that the behavioral task itself departs too much from true prism adaptation.

The nature of the fMRI experimental design itself is another key issue when investigating the neural correlates of prism adaptation. Typically, prism adaptation procedures involve three mandatorily successive steps: (1) pointing without prisms (baseline); (2) pointing with prisms (prism exposure); and (3) pointing after the removal of prisms. The difference in pointing error between steps 1 and 3 is the after-effect measure. Studies that depart from this sequence or that cycle through the sequences too quickly are likely to be unable to capture the neural mechanisms of sensorimotor realignment. In particular, behavioral studies have shown that adaptation after-effects develop slowly over successive trials of pointing under prism exposure and tend to stabilize and reach maximum magnitude after 50-60 exposure trials (Redding \& Wallace, 1993). In the pioneering positron emission tomography (PET) study reported by Clower and her colleagues (1996), the optical deviation was reversed (left to right) every five trials to maintain the participant in a state of on-going adaptation. A subsequent fMRI study used the same reversing sequence (Danckert et al., 2008). However, the activity reported in these studies is more likely to be associated with the fast strategic recalibration of the visuomotor transformation induced by prisms, which occurs mainly in the first few trials of exposure, than the slower sensorimotor realignment process, which develops slowly over more trials (Redding et al., 2005). Therefore, the optimal design for investigating the neural correlates of sensorimotor realignment would require at least 50 successive trials of pointing under exposure to the same prism direction in order to capture the slower realignment processes.

The optimal prism adaptation procedure is somewhat at odds with optimal fMRI study design. fMRI investigations of cognitive functions usually involve comparing BOLD responses during some task(s) of interest to those that occur during a highly comparable control task. There are test-retest effects that are specific to fMRI studies, such as habituationdependent dampening of the positive bold response (Thompson \& Spencer, 1966). Common ways to compensate for these time-based effects are to present several short blocks - or epochs - of each task across a single run, or to present the two conditions in two separate runs, the order of which is counterbalanced across participants. Manual pointing at targets viewed through neutral (non-shifting) lenses provides a good sham adaptation procedure, since this reproduces the visual and motor requirements of prism adaptation without inducing any strategic recalibration or sensorimotor realignment. However, investigating the neural correlates of true prism adaptation precludes the use of alternating blocks of sham and true prism adaptation, since this would interrupt the sustained stream of 50 exposure trials that is essential to induce sensorimotor realignment. Furthermore, periods of pointing under neutral vision that immediately follow prism adaptation evoke deadaptation: sensorimotor realignment from the adapted state back to normal alignment. Thus, any closed-loop pointing under neutral vision that follows prism adaptation is not a true sensorimotor control condition. Overall, the nature of prism adaptation precludes both the use of several short blocks of sham and real prism adaptation in alternating or random order within a single run, or counterbalancing the order of entire runs of sham and prism adaptation across participants. One option is to use a betweensubjects approach, in which one group undergoes real and the other sham prism adaptation. An alternative within-subjects approach is to include a long period of prism adaptation of at least 50 pointing movements preceded by a similar period of sham adaptation.

One way to circumvent the confounding order-dependent effect that such a design would introduce is to incorporate behavioral indices as parametric modulators that are used as independent predictors in the design matrix. In a previous event-related fMRI study looking for dynamic changes in brain activity during prism adaptation, the size of pointing errors was used to derive behavioral indices of error detection and error correction during CLP (Luauté et al., 2009). Identifying the neural correlates of sensorimotor realignment could be achieved by using intermittent OLP trials throughout the prism exposure period. From this, a behavioral index of the adaptation after-effect - the measure of true sensorimotor realignment - could be derived. Errors for CLP and OLP trials would together provide behavioral indices of the two major components of prism adaptation: strategic correction and sensorimotor realignment.

Our goal for the work presented here was to develop a prism adaptation system that would fulfill several requirements that are important for prism adaptation, while also allowing it to be performed in an fMRI environment. These 
requirements and the extent to which existing studies fulfill them are summarized in Table 1. Four existing studies have attempted to address the challenges of investigating the neural correlates of prism adaptation using fMRI (Chapman et al., 2010; Danckert et al., 2008; Küper et al., 2014; Luauté et al., 2009). While these pioneer studies have paved the way to investigate the neural correlates of various behaviors associated with prism adaptation, their designs could be further improved upon.

The first requirement was that participants should have direct vision of their hands and the targets. Viewing the hands and targets indirectly through a forward-projecting mirror system, as is often used in fMRI studies, introduces the confounds of additional visuospatial transformations above and beyond that involved in prism adaptation. Rather than using a mirror, an ideal system would instead enable participants to directly view the CLP and OLP targets, as well as their pointing hands during the second half of each CLP trial. Behavioral studies of prism adaptation treatment for hemispatial neglect typically provide visual feedback of the hand during the second (distal) half of the pointing movement ('concurrent' or 'continuous' feedback; Choe \& Welch, 1974; Heuer \& Hegele, 2008; Redding et al., 2005). This procedure provides both visual and proprioceptive feedback of the pointing movement, which is presumed to optimize generalization of the adaptation after-effect, although there is a paucity of direct evidence that this translates into greater neglect reduction than the commonly used alternative approach in which only the most distal $1-2 \mathrm{~cm}$ are visible ("terminal" feedback; Facchin, Daini, \& Toraldo, 2013).

The second requirement for a system to enable prism adaptation in an fMRI environment was that there should be the means to rapidly switch between prismatic vision, neutral vision, and occluded vision. This is essential if one wishes to dynamically switch between CLP and OLP trials, which is necessary for measuring the evolution of both error correction and adaptation after-effect and incorporating these behavioral indices into an analysis of BOLD signal changes. Third, it should be possible to switch between these three view types without any involvement of the participant (i.e., without the need for the participant to move a manipulandum from one eye to another, or the need to rely on the participant to follow an instruction to close their eyes). This helps the participant remain naïve as to which condition they are being exposed to, which, in healthy participants, produces a stronger adaptation after-effect (Michel et al., 2007). It avoids neural confounds associated with these intervening cognitive processes. It also ensures that the system is well suited for studies involving stroke patients, who may have difficulty following complex instructions while in the scanner.

We also considered it desirable to expose participants to prismatic vision binocularly. The phenomenon of binocular summation refers to the observation that the performance of neurologically healthy participants on many visual tasks is better when completed under binocular compared to monocular conditions (Blake \& Fox, 1973). This binocular benefit is greater than what would be predicted on the basis of statistical considerations alone (i.e., probability summation), suggesting that binocular summation reflects neural interaction between the signals from the eyes. Furthermore, monocular occlusion alters spatial perception in neglect patients (Smania, Fonte, Picelli, Gandolfi, \& Varalta, 2013; Walker, Young, \& Lincoln, 1996) and neurologically healthy participants (Roth, Lora, \& Heilman, 2002), and these effects may be different depending on which eye is occluded (Burtis, Williamson, Mishra, \& Heilman, 2014; Chen, Erdahl, \& Barrett, 2009; Roth et al., 2002). This could impact upon the interpretation of functional results of prism adaptation studies,

Table 1 Design requirements for fMRI-compatible prism adaptation and the extent to which these were fulfilled by existing studies

\begin{tabular}{|c|c|c|c|c|}
\hline Design requirement & Danckert et al. (2008) & Luauté et al. (2009) & Chapman et al. (2010) & Küper et al. (2014) \\
\hline Direct vision of the hand and pointing target & $\checkmark$ & $\checkmark$ & $x$ & $x$ \\
\hline Rapidly switch between prismatic/neutral/occluded vision & $x$ & $x$ & $x$ & $x$ \\
\hline $\begin{array}{l}\text { Switching between prismatic/neutral/occluded vision without } \\
\text { involvement of participant }\end{array}$ & $x$ & $x$ & $\checkmark$ & $\checkmark$ \\
\hline Binocular vision & $x$ & $x$ & $x$ & $x$ \\
\hline Use of hand for pointing rather than manipulandum & $\checkmark$ & $\checkmark$ & $x$ & $\checkmark$ \\
\hline Measurement of pointing errors during fMRI scanning & $x$ & $\checkmark$ & $\checkmark$ & $\checkmark$ \\
\hline $\begin{array}{l}\text { Measurement of both error correction } \\
\text { (“closed-loop pointing") and after-effect } \\
\text { ("open-loop pointing") }\end{array}$ & $x$ & $\checkmark / X^{*}$ & $\checkmark$ & $\checkmark / X^{*}$ \\
\hline Long periods ( $\geq 50$ consecutive trials) of prism exposure & $x(10$ trials $)$ & $x(24$ trials $)$ & $x(18$ trials $)$ & $x(30$ trials $)$ \\
\hline Prism adaptation contrasted against true sham adaptation & $x^{\dagger}$ & $\checkmark$ & $x^{\dagger}$ & $\checkmark$ \\
\hline
\end{tabular}


particularly when different eyes are used for the sham and prism exposure conditions (as in Chapman et al., 2010, and Luauté et al., 2009). Although binocular exposure to a prismatically-shifted visual field is the norm in non-imaging behavioral studies of prism adaptation, all existing fMRI studies of prism adaptation have used monocular viewing.

There were also several design requirements that we considered to be important for the performance and recording of pointing movements. It is important that participants reach out and touch the targets with their own hand rather than using a manipulandum. This is more ecological and better reflects the use of prism adaptation therapeutically. Using manipulanda instead could introduce further sensorimotor transformations over and above those involved in prism adaptation. Furthermore, an optimal design would be one that could be administered to both healthy controls and patient participants, and the latter group may have difficulties using a manipulandum.

Another design requirement was to be able to record the trial-by-trial pointing errors of the participants during the scanning run. This would allow for regressors in the functional imaging design that correlate with the evolution of pointing errors. It would be particularly advantageous to be able to measure both CLP and OLP errors, since these are indicators of different components of prism adaptation.

In the current paper we first describe the apparatus and methods that we created in order to address the design requirements outlined above. We then report behavioral (pointing) and head-movement data from a study that we conducted in order to validate our fMRI-compatible prism adaptation system.

\section{Development and technical specifications}

\section{Design and construction}

Prism adaptation visor We constructed a prism adaptation "visor" that could be fixed to a head coil in front of the participants' line of vision (Fig. 1). The operation and visual effect of the visor are demonstrated in Supplemental Videos $1-3$, available online. The visor is made of plastic and has a $20 \mathrm{~cm}$ wide $\times 4 \mathrm{~cm}$ high viewing window. For prism exposure, a single 15 diopter Fresnel prism sheet giving a rightward prismatic shift of $7.7^{\circ}$ (RHK Japan Inc, Tokyo) can be lowered into the window. A sheet of clear Perspex that produces no lateral shift in vision can be used for sham adaptation. The prism and neutral sheets can be raised and lowered into the window. The raising and lowering of the sheets is

\footnotetext{
${ }^{1} 10^{\circ}$ is the most typical prism strength for rehabilitation of neglect. We were able to procure prism sheets that induced $7.7^{\circ}$ and $12.5^{\circ}$ shifts. In pilot testing we found that the $12.5^{\circ}$ prism sheet resulted in pointing errors that exceeded the area of our touch screen (i.e., participants' pointing endpoints during the first trials of prism exposure were outside the active surface of the touch screen). We therefore used $7.7^{\circ}$ prisms in our prism adaptation visor.
}

controlled by an experimenter by means of strings that extend through the scanner bore to the back of the scanner. The visor also includes an occluding panel that can be raised and lowered in the same manner to block the participant's vision for OLP trials and when changing between prism adaptation and neutral pointing (so as to keep the participant naïve as to the present condition). All other directions of vision outside this window can be occluded using fabric such that the participants' field of view consists only of that which they can see through the window of the visor.

Stimulus presentation and response collection We constructed a touchscreen device for target presentation and measurement of terminal pointing errors. The device consists of a Keytech Magictouch touchscreen with a responsive surface $22 \mathrm{~cm}$ high $\times$ $30 \mathrm{~cm}$ wide that is set into a wooden frame. A $3 \mathrm{~mm}$ thick plastic insert affixed to the back surface of the screen renders the screen opaque. The targets for prism adaptation are three LEDs fixed to the back of the screen through small holes $(\mathrm{d}=1 \mathrm{~mm})$ in the plastic backing: a blue LED at the center of the screen and two red LEDs $5 \mathrm{~cm}$ to the left and right of the center of the screen. All targets are at a height of $11 \mathrm{~cm}$ from the bottom of the screen. Awhite piece of paper between the plastic insert and the touchscreen ensures the LEDs are not visible to the participant unless illuminated. The touchscreen is held in position over the participant's lap by a wooden frame that spans the width of the scanner bed and is secured using industrial tape. We determined that there was potential for the edge of the wooden frame to fall within some participants' field of vision, which could provide a spatial cue to the different visual conditions. To eliminate this cue we covered the frame with white paper toweling to render it effectively invisible in the semi-illuminated scanning environment.

We use Presentation software (www.neurobs.com) to control the onset of the LEDs via the parallel port and to record the touch-screen responses. The wires connecting the touchscreen and the LEDs to the computer are insulated with aluminum foil to prevent radiofrequency artifact.

Participant placement The participant's head must be tilted toward their body in order for them to direct their gaze through the visor. To achieve this we adapted the scanning environment based on existing studies that have employed direct vision of the hands, arms, and reaching space (e.g., Culham et al., 2003; Gallivan, McLean, Valyear, \& Culham, 2013; Gallivan, McLean, Flanagan, \& Culham, 2013; Rossit, McAdam, Mclean, Goodale, \& Culham, 2013). We recommend using a thin bed pad that provides sufficient cushioning for comfort but allows the participant's body to be as low as possible relative to the head coil, facilitating head tilt. We also recommend using a large head-coil that is inclined relative to the scanner bed. In our configuration, we use a piece of wood positioned under the back part of the head coil to achieve this tilt. A custom-made $15 \mathrm{~cm}$ coil cable accommodates the 


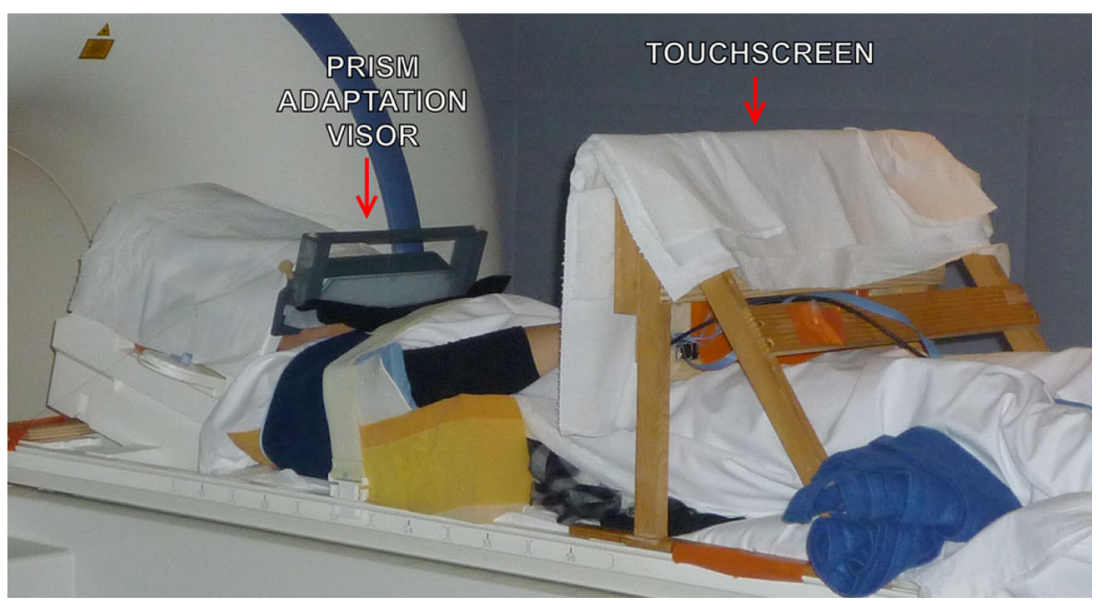

Fig. 1 Configuration for performing prism adaptation within the fMRI environment. Paper toweling around the head coil and black fabric along the bottom of the prism adaptation visor restricts the participant's field of view to that which can be seen through the window of the visor. The right arm is stabilized by cushions placed under and around the arm, and by straps securing the upper arm to the torso. Paper toweling is placed around the frame of the touchscreen to prevent it from providing a

greater distance between the coil and the scanner bed. Using a large head-coil also provides sufficient space for the participant to further tilt their head within the coil. With these three steps (using a thin bed pad, inclining the head coil relative to the scanner bed, and inclining the head within the head coil) we achieve head tilts of $15-20^{\circ}$.

In order to minimize movement of the head during target pointing, the pointing arm should be positioned in such a way as to limit all arm movements to below the elbow. The upper arm can be supported in a raised position with foam cushions and strapped firmly and comfortably to the participant's body. In our configuration we achieve this through means of a large wedge-shaped cushion positioned under the upper arm, a "half-cast" in which the upper arm was placed, and a broad physiotherapists strap that held the upper arm close to the torso. This arrangement allows the participant to limit arm movements to the wrist and elbow.

The touch-screen is placed vertically over the participant's lap at whichever depth (relative to their head and feet) enables them to comfortably reach all of the targets at arm's length. Accommodating for differences in arm length in this way reduces the likelihood of head movements that could be caused by a participant straining to reach a target that is too distant or distorting their posture to reach a target that is too near. Although this procedure introduces inter-individual variability in the distance between the touch-screen and the participant's eyes, this is a minor disadvantage compared to potential movement artifacts. Pointing errors can be converted from centimeters to degrees of visual angle by measuring the distance between each participant's eyes and the touch screen (and doing the following computation: pointing error in degrees $=\tan ^{-1}$ (pointing error in centimeters/distance between eyes and touch screen in centimeters). frame of reference that would indicate shifting spatial reference frames between prismatic and neutral vision. During the experiment fabric was placed over the participant's torso and upper arm (not pictured) to ensure that their arm was only visible during the second half of the pointing movement as per typical prism adaptation procedures (Redding et al., 2005)

\section{Evaluation of prism adaptation set-up}

Using the materials and configuration described above, two groups of older neurologically healthy participants and right hemisphere lesioned patients underwent prism adaptation in the MRI scanner. Here we present behavioral (i.e. OLP and CLP error) data as evidence that the apparatus we have designed provides a good solution for measuring prism adaptation (and in particular sensorimotor realignment) in the fMRI environment.

\section{Materials and methods}

Participants Twelve stroke patients were recruited through the department of neurorehabilitation in Lyon University Hospital and 18 neurologically healthy control participants were recruited through advertisements and word of mouth. All participants gave informed consent to a research protocol that was approved by the local ethics committee according to the Declaration of Helsinki and French law.

Inclusion criteria for patient participants were that the person had suffered a first-time stroke to the right-hemisphere and had shown neglect at some point subsequent to their stroke. Exclusion criteria for both patient and control participants were the presence of: (1) a history of neurological injury or illness (controls), or neurological injury or illness other than a single right-hemisphere stroke (patients), (2) contraindications to undergoing MRI scanning, (3) visual or motor impairments preventing the performance of prism adaptation, and (4) factors limiting the participant's capacity to provide informed 
consent, such as cognitive impairment or insufficient fluency in English or French.

Of the tested participants, one patient was excluded because a piece of occluding material became dislodged from the head coil early in the testing session and prevented him from seeing all of the targets. One control participant was also excluded because it was discovered after the testing session that she had been unable to comfortably reach the leftmost target. This participant showed no behavioral effects of prism adaptation, suggesting that the physical constraints of the testing environment prevented her from performing prism adaptation normally. The pointing data from the remaining 11 patients (two females, mean age $=52, S E M=3.6$ ) and 17 control participants (nine females, mean age $=61, S E M=3.2$ ) were analyzed.

Clinical information for the patients is provided in Table 2. Neurological examinations showed that at the time of study participation eight patients had hemiplegia, ten showed some sensory loss, and eight showed some degree of visual field loss. Patients RH01, RH03, and RH09 demonstrated neglect at the time of testing (defined as at least two more targets omissions on the left of the page compared to the right of the page on the Bell's cancellation test; Azouvi et al., 2002; Gauthier, Dehaut, \& Joanette, 1989).

A lesion composite image is shown in Fig. 2. Each patient's lesion was manually traced from the structural scan using FSLview software and validated by a neurologist. The composite image was created using MRIcron software (Rorden, Karnath, \& Bonilha, 2007). The region of maximal overlap [MNI $(x, y, z)=36,-24,18]$ is located in the secondary somatosensory cortex, in the parietal operculum on the ceiling of the lateral sulcus.

Procedure Participants completed one run of sham adaptation followed by one run of real prism adaptation while in the scanner. The general procedure was as follows: Before entering the scanner room all participants were instructed on how to perform the CLP and OLP movements and had the opportunity to ask questions. They then entered the scanner room and were positioned as described above. A strap was secured over the participant's forehead to assist them in stabilizing their head, and headphones were placed over their ears. Once the touch screen and prism adaptation visor were positioned an emergency call button was placed near the participant's left hand and the task instructions were explained a second time. To ensure that they had a high degree of familiarity with the task, each participant underwent a practice "run" immediately prior to being inserted into the scanner. The practice run was identical to the sham run (see below) except that it was performed before the scanner bed was inserted into the scanner, and the participants received verbal feedback from an experimenter and additional instructions if necessary.

Experimental paradigm Figure 3 provides an overview of the behavioral task that was performed during the session. In two consecutive runs, participants underwent sham (run 1) and then prism (run 2) exposure using their right hand. For this, they performed CLP movement to targets viewed through the neutral sheet (sham run) or the prism sheet (prism run) in which their hand was visible for the second half of the pointing movement. They also performed OLP movements in which they viewed targets though the neutral sheet that were then occluded before they pointed to the location of the target (sham and prism run; see Supplemental Video 3 online).

Each run consisted of ten blocks each of CLP and OLP in alternating order. There were six pointing trials per block, resulting in a total of 60 CLP and 60 OLP trials per run. Each CLP trial began with the illumination of the left or right

Table 2 Clinical information for the patient participants

\begin{tabular}{|c|c|c|c|c|c|c|}
\hline Patient & $\begin{array}{l}\text { Age } \\
\text { (years) }\end{array}$ & Sex & $\begin{array}{l}\text { Chronicity } \\
\text { (weeks) }\end{array}$ & $\begin{array}{l}\text { Stroke } \\
\text { type }\end{array}$ & $\begin{array}{l}\text { Neurological } \\
\text { test outcome }\end{array}$ & $\begin{array}{l}\text { Bells } \\
\text { Cancellation } \\
\text { Test (Total } \\
\text { omissions) }\end{array}$ \\
\hline RH01 & 39 & M & 103 & $\mathrm{H}$ & none & 2 \\
\hline RH02 & 42 & $\mathrm{~F}$ & 136 & $\mathrm{H}$ & $\mathrm{He}, \mathrm{S}, \mathrm{V}$ & 0 \\
\hline RH03 & 47 & M & 14 & I & $\mathrm{He}, \mathrm{S}, \mathrm{V}$ & 9 \\
\hline RH04 & 69 & M & 100 & I & $\mathrm{He}, \mathrm{S}, \mathrm{V}$ & 3 \\
\hline RH05 & 56 & M & 139 & $\mathrm{IH}$ & $\mathrm{He}, \mathrm{S}, \mathrm{V}$ & 0 \\
\hline RH06 & 66 & M & 37 & $\mathrm{IH}$ & $\mathrm{S}, \mathrm{V}$ & 1 \\
\hline RH07 & 63 & M & 196 & I & $\mathrm{S}, \mathrm{V}$ & 5 \\
\hline RH08 & 59 & M & 43 & I & $\mathrm{He}, \mathrm{S}, \mathrm{V}$ & 2 \\
\hline RH09 & 47 & $\mathrm{~F}$ & 26 & I & $\mathrm{He}, \mathrm{S}, \mathrm{V}$ & 9 \\
\hline RH10 & 42 & M & 77 & I & $\mathrm{He}, \mathrm{S}$ & 1 \\
\hline RH11 & 50 & M & 26 & $\mathrm{H}$ & $\mathrm{He}, \mathrm{S}, \mathrm{V}$ & 4 \\
\hline
\end{tabular}

$M$ male, $F$ female, $H$ hemorrhagic, $I$ ischemic, $H e$ hemiplegia, $S$ sensory loss, $V$ visual field loss 


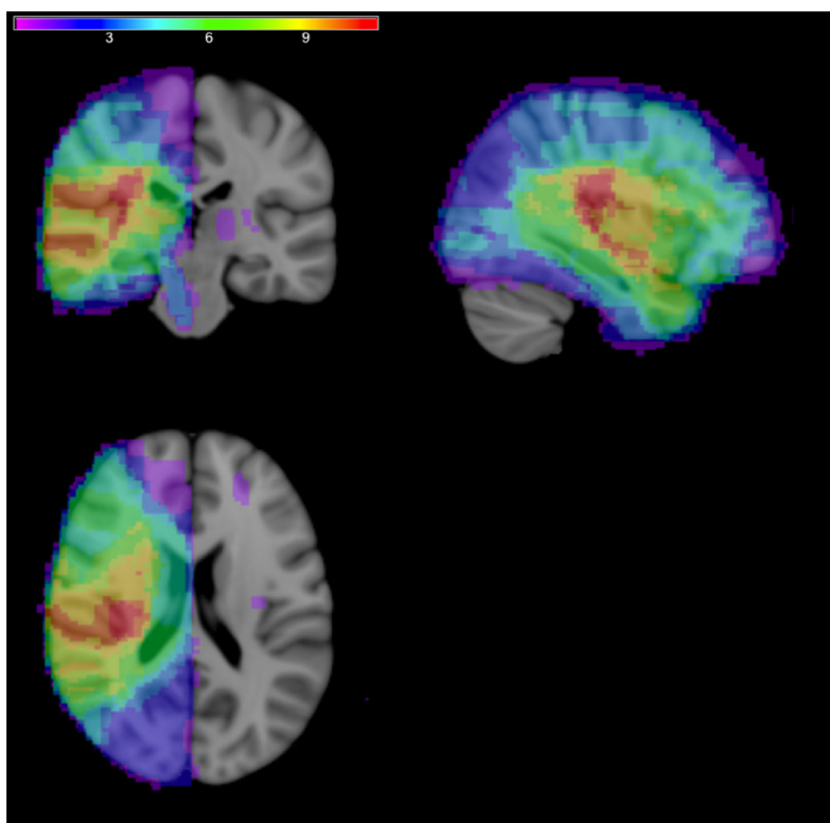

Fig. 2 Composite image of the lesions of the 11 patients. The image is shown overlaid onto axial, coronal and sagittal slices of the MNI T1 standard brain. In line with radiological convention, the sagittal and coronal views are depicted as viewed from below with right-hemisphere lesions on the left side of the image

red LED, viewed through the neutral sheet (practice and sham runs) or the prism sheet (prism run). The participants were instructed to touch the light using a smooth, rapid motion and then return their hand to rest on their torso. They were able to see their pointing finger and hand for the second half of each pointing movement. The LED extinguished upon the participant touching the touch screen. Three left trials and three right trials were presented in pseudorandom order within each CLP block, and individual CLP trials were separated by $6.5 \pm 1 \mathrm{~s}$.

Each OLP trial began with the illumination of the blue LED in the center of the screen viewed through the neutral sheet (all runs) after which the experimenter lowered the occluding panel. From the participant's perspective the lowering of the occluding panel made it appear as though the target had disappeared, which is important because sensorimotor realignment is maximized when participants are unaware of the spatial distortions caused by the prism (Michel et al., 2007). Participants were instructed to reach out to touch the remembered location of the target and return their hand to rest on their torso. The experimenter raised the occluding panel once the participant's hand had returned to their torso. Each OLP trial was separated by $6.5 \pm 1 \mathrm{~s}$.

Between each CLP and OLP block the experimenter lowered the occluding panel to block the participant's vision. During the prism run this enabled the neutral and prism sheets to be switched without the participant's awareness (see Supplemental Videos 1 and 2 online). No switch was made during the practice or sham runs as the sham sheet was used throughout the entire run, however the occluding panel was always lowered to mark a pause in the transition between successive CLP and OLP blocks, and to ensure that the procedures for the sham and prism runs were identical. The occluding panel was raised again once the sheets (prism/neutral) had been switched $(\sim 1 \mathrm{~s})$. The inter-block interval was $7.5 \pm 1 \mathrm{~s}$.

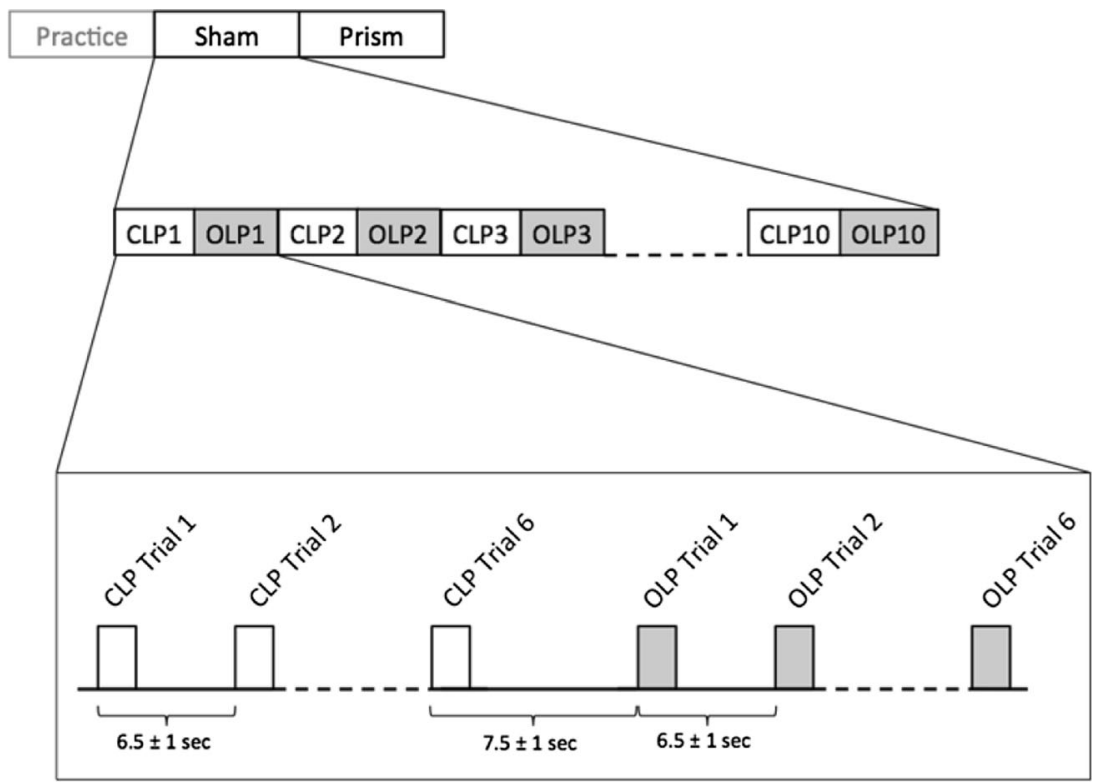

Fig. 3 Overview of the behavioral task. The top row indicates the run order, the middle row indicates the block order within each run, and the bottom row indicates the trial timings. The practice, sham, and prism runs were identical. Each CLP and OLP block consisted of six individual trials. $C L P$ closed-loop pointing, $O L P$ open-loop pointing 
For each CLP and OLP trial the coordinates of the touched location were converted to lateral pointing error with respect to the target and converted from centimeters to degrees of visual angle.

Imaging protocol Imaging was performed at the Centre d'Etude et de Recherche Multimodal et Pluridisciplinaire (CERMEP) in Lyon using a Siemens Magnetom Sonata 1.5 T scanner with a circularly polarized head coil with two integrated preamplifiers. Anatomical images were acquired with a MPR T1 sequence (176 slices, FOV $=256 \mathrm{~mm}, \mathrm{TR} / \mathrm{TE} /$ flip angle $=1.97 / 3.93 / 15^{\circ}, 1.0 \times$ $1.0 \times 1.0 \mathrm{~mm}$ voxels) taking 8.26 minutes. Functional MRI of the whole brain was acquired in the axial plane ( 26 slices, $\mathrm{FOV}=220$ $\times 220 \mathrm{~mm}^{2}, \mathrm{TR} / \mathrm{TE} / \mathrm{flip}$ angle $=2,500 \mathrm{~ms} / 60 \mathrm{~ms} / 90^{\circ}, 3.4 \times 3.4 \times$ $5.0 \mathrm{~mm}$ voxels). Each of the functional runs took $12.35 \mathrm{~min}$.

Head motion parameters were measured using MCFLIRT (Jenkinson, Bannister, Brady, \& Smith, 2002) part of FSL [FMRIB's Software Library, www.fmrib.ox.ac.uk/fsl (Smith et al., 2004)]. We report head motion parameters as indicators of the efficacy of our prism adaptation configuration in enabling true prism adaptation while keeping head movements within reasonable limits.

Functional brain imaging data are not reported here, since the purpose of this report is to present and validate the experimental apparatus.

\section{Results}

Pointing error data In order to test for effective prism adaptation, each participant's CLP and OLP errors were

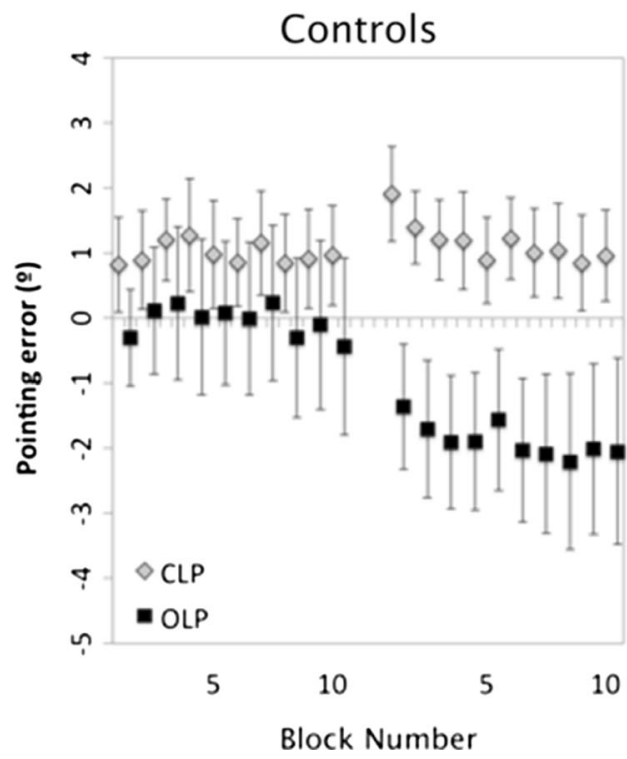

Fig. 4 Closed-loop pointing (CLP) and open-loop pointing (OLP) errors of the control (left panel) and patient (right panel) participants across the ten blocks of the sham and prism runs. Pointing errors are measured in degrees of horizontal displacement from the target location. Positive averaged across each block of six trials separately for the sham and prism run and pooled for analysis. The evolution of CLP and OLP errors across the different blocks and run is represented separately for controls and patients in Fig. 4. The mean CLP and OLP errors were subjected to separate repeated-measures ANOVAs with Run (sham or prism) and Block $(1,2,3,4,5,6,7,8,9$, or 10) as within-subjects factors and Group (patient or control) as a between-subjects factor. Both datasets violated the assumption of sphericity $(W \mathrm{~s}<0.008, p \mathrm{~s}<.001)$ so Greenhouse-Geisser-corrections were used.

The analysis of CLP errors revealed a significant Run $\times$ Block interaction $\left[F(9,234)=3.8, p<.01, \eta_{\text {partial }}^{2}=\right.$ 0.13 ] reflecting the fact that pointing errors were initially right-shifted in the prism run and gradually returned to baseline accuracy as a function of adaptation, whereas no such trend occurred in the sham run (Fig. 4). A priori paired t-tests were conducted to compare the mean CLP of each block of the sham run to the equivalent block of the prism run (block 1 of the sham run was compared to block 1 of the prism run and so forth). The CLP error for the first block of the prism run $(M=2.06, S E M=0.35)$ was $1.34^{\circ}$ rightward of the CLP error for the first block of the sham run $[M=0.72, S E M=0.23 ; t(27)=3.7, p<$ .005]. There was also a trend toward more rightward CLP errors for the second block of the prism run $(M=1.36$, $S E M=0.41)$ compared to the second block of the sham run $(M=0.83, S E M=0.32)$, although this difference did not reach statistical significance $[t(27)=1.9, p=.067]$. None of the pairwise comparisons for later blocks were significant $(t \mathrm{~s}<2.0, p \mathrm{~s}>.053)$. No main effects and no

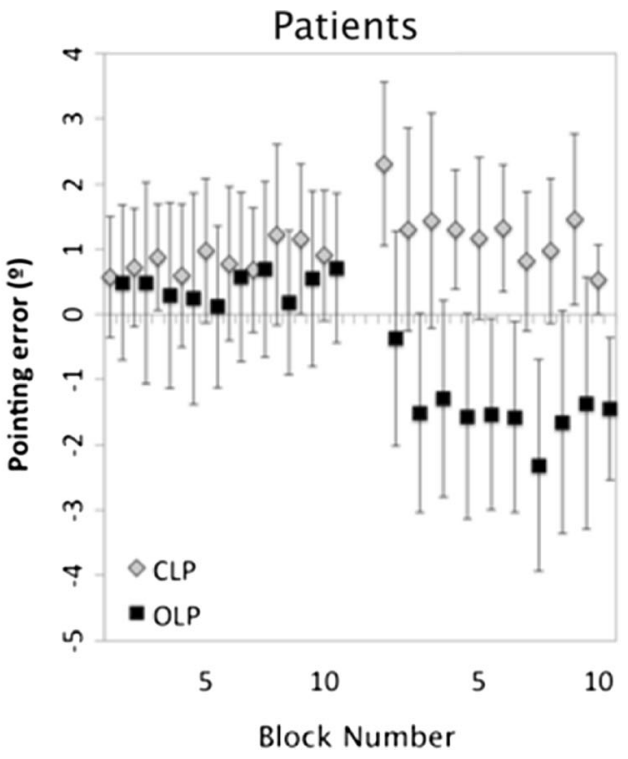

values indicate rightward errors and negative errors indicate leftward errors relative to the true target position. Error bars represent 95\% confidence intervals around the means 
other interactions aside from the Run $\times$ Block interaction were significant in the analysis of CLP errors $\left(F_{\mathrm{S}}<1.3\right.$, $p \mathrm{~s}>.26)$, including no main effect of Group $[F(1,26)=$ $0.001, p=.97]$.

The analysis of OLP errors revealed a significant main effect ofRun $\left[F(1,26)=52.1, p<.005, \eta_{\text {partial }}^{2}=0.67\right]$ which reflected significantly leftward shifted pointing errors (sensorimotor recalibration) during the prism run $(M=-1.7, S E M=0.49)$ compared to the sham run $(M=0.2, S E M=0.47)$, consistent with an adaptation after-effect of $1.9^{\circ}$, or $25 \%$ of the magnitude of the prismatic shift. A priori paired t-tests were conducted comparing the mean OLP of each block of the sham run to the equivalent block of the prism run, and revealed that for every pairwise comparison the pointing error for the prism condition was significantly leftward of the sham condition $(t \mathrm{~s}>0.36, p \mathrm{~s}<.003$; see Fig. 4). There were no other significant main effects or interactions $\left(F_{\mathrm{s}}<2.1, p \mathrm{~s}>.1\right)$, including no main effect of Group $[F(1,26)=0.23, p=.63]$.

Head movement data The median scores for mean relative displacement $(\mathrm{mm})$ values for the control group were 0.08 (range: $0.06,0.41)$ during the sham run and 0.1 during the prism run (range: $0.04,0.33$ ). The medians for the patient group were 0.14 (range: $0.09,0.83$ ) during the sham run and 0.18 (range: $0.09,2.01$ ) during the prism run. Neither group showed a significant difference in mean relative displacement for the sham compared to the prism runs $(Z s<1.6, p s>.11)$. A rule of thumb is that mean relative displacements greater than $0.5 \mathrm{~mm}$ should be considered problematic (Power et al., 2014). By this criterion, potentially problematic head movements were shown by one patient during both the sham and prism runs (mean relative displacements $=0.83$ and 2.01 respectively), and one patient during the prism run only (mean relative displacements $=0.55$ ). Thus, the majority of our participants were able to maintain sufficient head stability to satisfy standard quality limits for fMRI studies.

\section{Discussion}

Our goal was to develop a system for performing prism adaptation in an fMRI environment. A few previous studies have already succeeded in performing functional neuroimaging of prism adaptation, fulfilling some to most of the criteria we identified as the possible ideal combination of design and apparatus/procedures. In trying to fulfill the whole of these criteria, we developed an apparatus and procedures to deliver prism adaptation in a way that is closely aligned to that from both classic and contemporary studies (Harris, 1965; Redding et al., 2005). Our behavioral results show the presence of two patterns in the CLP and OLP errors of the participants that are characteristic of prism adaptation. First, compared to the sham run CLP errors in the prism run showed an initial mean rightward deviation of $1.34^{\circ}(\mathrm{SEM}=0.36)$ that was not present during the remaining nine blocks. This is consistent with the direct effects of prism exposure (that is, the pointing errors that occur during the initial exposure to rightward-deviating prisms which reduce within the first few trials). Second, OLP errors in the prism run were significantly leftward relative to those in the sham run, indicating an adaptation after-effect of $1.9^{\circ}(\mathrm{SEM}=0.25)$, or $25 \%$ of the prismatic shift. This aftereffect is comparable to those reported in existing non-imaging studies that used OLP for after-effect measurement in which after-effects typically have a magnitude that is $25-40 \%$ of the prismatic deviation (Bultitude, Van der Stigchel, \& Nijboer, 2013; Bultitude \& Woods, 2010; Girardi, McIntosh, Michel, Vallar, \& Rossetti, 2004; Loftus et al., 2009; Michel et al., 2007). We can conclude, therefore, that our system enables the performance of true prism adaptation and recording of both CLP and OLP errors in the AMRI environment.

Head movements were within $0.5 \mathrm{~mm}$ of mean relative displacement for all but two participants. There are no absolute rules about how much head movement can be tolerated in fMRI studies. A study involving highly experienced participants performing perceptual tasks that require minimal motor responses (e.g., button presses) might expect mean relative displacements of 0.1 or $0.2 \mathrm{~mm}$. In contrast, head movements for at least some participants are likely to exceed this amount in studies such as the current one that involve naïve older controls, patients, and a task that requires arm movements. Nonetheless, the amount of movement that we have reported is comparable to other studies that involve arm (or leg) movements within the scanner (Gallivan, McLean, Flanagan, et al., 2013; Singhal, Monaco, Kaufman, \& Culham, 2013) and is within the limits of what could reasonably be corrected by motion correction algorithms.

No formal assessment was made of comfort levels during the current study; however, informal discussion revealed that the procedure was well tolerated by both patients and healthy volunteers in that any discomfort that was reported was not more than that which would be expected in a normal fMRI study in which participants lie flat and view stimuli by means of a mirror.

A crucial reason to perform a functional imaging study during prism adaptation is to search for the neural mechanisms supporting the generalization of simple sensorimotor adaptation to the reconfiguration of higher-level spatial representations. Although the generalization of prim adaptation aftereffects require a different design with measures of spatial cognition, future attempts scrutinizing this important issue might benefit from the present experiment to find ways of avoiding pitfalls regarding prism adaptation procedure in a MRI scanner. These could be further adapted to accommodate specific study requirements. For example the prism adaptation visor could be controlled using a MR-compatible pneumatic mechanism in order to achieve precise timing of transitions between prismatic, neutral and occluded vision, and to eliminate the 
need for an experimenter to be in close proximity to the scanner.

\section{Conclusions}

In summary, we have described an apparatus and protocols for administering prism adaptation in the fMRI environment. We constructed a new prism adaptation visor that enables rapid switching between binocular prismatic, neutral and occluded vision in the confined space of the scanner bore and without the involvement of the participant. We developed a configuration in which both patients and neurologically healthy control participants could point to visual targets with their own hand for 50 or more exposure trials and with direct vision of their hand and the targets. The touchscreen system captures pointing errors made by the participants during both CLP and OLP runs which, by alternating these blocks, enables the gradual evolution of error correction and sensorimotor realignment to be measured separately. This should aid future fMRI studies to distinguish the neural correlates of error detection/correction and sensorimotor realignment. Finally, we have presented behavioral and head movement data that validate these methods by generating comparable data quality to that observed in existing non-imaging studies of prism adaptation. Our data also show comparable performance of the patients and control participants when they performed prism adaptation with these methods and apparatus. The apparatus and design considerations we outline could be used to guide future studies investigating the neural correlates of sensorimotor realignment during prism adaptation.

Author note This study was supported by grants from FRM (Fondation pour la Recherche Medicale) and the James S. McDonnell Scholar Award to AF, and was performed within the framework of the LABEX CORTEX (ANR-11-LABX-0042) of Université de Lyon. JO'S was supported by a Royal Society Dorothy Hodgkin Fellowship. The authors wish to thank Jody Culham and Adam McLean for their advice on achieving direct vision of the hands in an fMRI environment, Paul Downing for helpful discussions about the manuscript and Frederic Volland for building the set-up. The authors declare that there is no conflict of interest regarding the publication of this paper.

Open Access This article is distributed under the terms of the Creative Commons Attribution 4.0 International License (http:// creativecommons.org/licenses/by/4.0/), which permits unrestricted use, distribution, and reproduction in any medium, provided you give appropriate credit to the original author(s) and the source, provide a link to the Creative Commons license, and indicate if changes were made.

\section{References}

Azouvi, P., Samuel, C., Louis-Dreyfus, A., Bernati, T., Bartolomeo, P., Beis, J.-M., Rousseaux, M. (2002). Sensitivity of clinical and behavioural tests of spatial neglect after right hemisphere stroke.
Journal of Neurology, Neurosurgery, and Psychiatry, 73(2), 160 166. doi: 10.1136/jnnp.73.2.160

Berberovic, N., \& Mattingley, J. B. (2003). Effects of prismatic adaptation on judgements of spatial extent in peripersonal and extrapersonal space. Neuropsychologia, 41(4), 493-503.

Blake, R., \& Fox, R. (1973). The psychophysical inquiry into binocular summation. Perception \& Psychophysics, 14(1), 161-185. doi:10.3758/BF03198631

Bultitude, J. H., Van der Stigchel, S., \& Nijboer, T. C. W. (2013). Prism adaptation alters spatial remapping in healthy individuals: Evidence from double-step saccades. Cortex, 49(3), 759-770. doi:10.1016/j. cortex.2012.01.008

Bultitude, J. H., \& Woods, J. M. (2010). Adaptation to leftward-shifting prisms reduces the global processing bias of healthy individuals. Neuropsychologia, 48(6), 1750-1756. doi:10.1016/j. neuropsychologia.2010.02.024

Burtis, D. B., Williamson, J. B., Mishra, M., \& Heilman, K. M. (2014). The blindside: Impact of monocular occlusion on spatial attention. Journal of Clinical and Experimental Neuropsychology, 35(3), 291297. doi:10.1080/13803395.2013.770824

Chapman, H. L., Eramudugolla, R., Gavrilescu, M., Strudwick, M. W., Loftus, A., Cunnington, R., \& Mattingley, J. B. (2010). Neural mechanisms underlying spatial realignment during adaptation to optical wedge prisms. Neuropsychologia, 48(9), 2595-2601. doi:10.1016/j.neuropsychologia.2010.05.006

Chen, P., Erdahl, L., \& Barrett, A. M. (2009). Monocular patching may induce ipsilateral "where" spatial bias. Neuropsychologia, 47(3), 711-716. doi:10.1016/j.neuropsychologia.2008.11.022

Choe, C. S., \& Welch, R. B. (1974). Variables affecting the intermanual transfer and decay of prism adaptation. Journal of Experimental Psychology, 102(6), 1076-1084.

Clower, D., Hoffman, J., Votaw, J., Faber, R., Woods, R., \& Alexander, G. (1996). Role of the posterior parietal cortex in the recalibration of visually guided reaching. Nature, 383, 618-621.

Culham, J. C., Danckert, S. L., DeSouza, J. F. X., Gati, J. S., Menon, R. S., \& Goodale, M. A. (2003). Visually guided grasping produces fMRI activation in dorsal but not ventral stream brain areas. Experimental Brain Research, 153(2), 180-189. doi:10.1007/s00221-003-1591-5

Danckert, J. A., Ferber, S., \& Goodale, M. A. (2008). Direct effects of prismatic lenses on visuomotor control: An event-related functional MRI study. European Journal of Neuroscience, 28(8), 1696-1704. doi:10.1111/j.1460-9568.2008.06460

Facchin, A., Daini, R., \& Toraldo, A. (2013). Prismatic adaptation in the rehabilitation of neglect patients: Does the specific procedure matter? Frontiers in Human Neuroscience, 7(April), 137. doi:10.3389/fnhum.2013.00137

Frassinetti, F., Angeli, V., Meneghello, F., Avanzi, S., \& Ladavas, E. (2002). Long-lasting amelioration of visuospatial neglect by prism adaptation. Brain, 125(Pt 3), 608-623.

Gallivan, J. P., McLean, D. A., Flanagan, J. R., \& Culham, J. C. (2013). Where one hand meets the other: Limb-specific and action-dependent movement plans decoded from preparatory signals in single human frontoparietal brain areas. The Journal of Neuroscience, 33(5), 19912008. doi:10.1523/JNEUROSCI.0541-12.2013

Gallivan, J. P., McLean, A. D., Valyear, K. F., \& Culham, J. C. (2013). Decoding the neural mechanisms of human tool use. eLife, 2013(2), 1-29. doi:10.7554/eLife.00425

Gauthier, L., Dehaut, F., \& Joanette, Y. (1989). The Bells Test: A quantitative and qualitative test for visual neglect. International Journal of Clinical Neuropsychology, 11(2), 49-54.

Girardi, M., McIntosh, R. D., Michel, C., Vallar, G., \& Rossetti, Y. (2004). Sensorimotor effects on central space representation: Prism adaptation influences haptic and visual representations in normal subjects. Neuropsychologia, 42(11), 1477-1487. doi:10.1016/j. neuropsychologia.2004.03.008 
Grootoonk, S., Hutton, C., Ashburner, J., Howseman, A., Josephs, O., Rees, G., ... Turner, R. (2000). Characterization and correction of interpolation effects in the realignment of fMRI time series. NeuroImage, 11(1), 49-57. doi:10.1006/nimg.1999.0515

Harris, C. S. (1965). Perceptual adaptation to inverted, reversed, and displaced vision. Psychological Review, 72, 419-444.

Hay, J. C., Langdon, B., \& Pick, H. L. (1971). Spatial parameters of eyehand adaptation to optical distortion. Journal of Experimental Psychology, 91(1), 11-17.

Hay, J. C., \& Pick, H. L. (1966). Visual and proprioceptive adaptation to optical displacement of the visual stimulus. Journal of Experimental Psychology, 71(1), 150-158. doi:10.1037/h0022611

Heilman, K. M., Valenstein, E., \& Watson, R. T. (2000). Neglect and related disorders. Seminars in Neurology, 20(4), 463-470.

Held, R., \& Freedman, S. J. (1963). Plasticity in human sensorimotor control. Science, 142(3591), 455-462.

Held, R., \& Mikaelian, H. (1964). Motor-sensory feedback versus need in adaptation to rearrangement. Perceptual and Motor Skills, 18, 685688.

Heuer, H., \& Hegele, M. (2008). Constraints on visuo-motor adaptation depend on the type of visual feedback during practice. Experimental Brain Research, 185(1), 101-110. doi:10.1007/s00221-007-1135-5

Jenkinson, M., Bannister, P., Brady, M., \& Smith, S. (2002). Improved optimization for the robust and accurate linear registration and motion correction of brain images. NeuroImage, 17(2), 825-841.

Küper, M., Wünnemann, M. J. S., Thürling, M., Stefanescu, R. M., Maderwald, S., Elles, H. G., ... Timmann, D. (2014). Activation of the cerebellar cortex and the dentate nucleus in a prism adaptation fMRI study. Human Brain Mapping, 35(4), 1574-1586. doi. 10.1002/hbm.22274

Liao, R., McKeown, M. J., \& Krolik, J. L. (2006). Isolation and minimization of head motion-induced signal variations in fMRI data using independent component analysis. Magnetic Resonance in Medicine, 55(6), 1396-1413. doi:10.1002/mrm.20893

Loftus, A., Nicholls, M. E. R., Mattingley, J. B., \& Bradshaw, J. L. J. (2008). Left to right: Representational biases for numbers and the effect of visuomotor adaptation. Cognition, 107(3), 1048-1058. doi:10.1016/j.cognition.2007.09.007

Loftus, A., Vijayakumar, N., \& Nicholls, M. E. R. (2009). Prism adaptation overcomes pseudoneglect for the greyscales task. Cortex, 45(4), 537-543. doi:10.1016/j.cortex.2007.12.011

Luauté, J., Halligan, P., Rode, G., Jacquin-Courtois, S., \& Boisson, D. (2006). Prism adaptation first among equals in alleviating left neglect: A review. Restorative Neurology \& Neuroscience, 24(4-6), 409-418.

Luauté, J., Jacquin-Courtois, S., O'Shea, J., Christophe, L., Rode, G., Boisson, D., \& Rossetti, Y. (2012). Left-deviating prism adaptation in left neglect patient: Reflexions on a negative result. Neural Plasticity, 2012, 718604. doi:10.1155/2012/718604

Luauté, J., Schwartz, S., Rossetti, Y., Spiridon, M., Rode, G., Boisson, D., \& Vuilleumier, P. (2009). Dynamic changes in brain activity during prism adaptation. The Journal of Neuroscience, 29(1), 169-178. doi:10.1523/JNEUROSCI.3054-08.2009

Michel, C., Pisella, L., Halligan, P., Luauté, J., Rode, G., Boisson, D., \& Rossetti, Y. (2003). Simulating unilateral neglect in normals using prism adaptation: Implications for theory. Neuropsychologia, 41(1), 25-39.

Michel, C., Pisella, L., Prablanc, C., Rode, G., \& Rossetti, Y. (2007). Enhancing visuomotor adaptation by reducing error signals: Single-step (aware) versus multiple-step (unaware) exposure to wedge prisms. Journal of Cognitive Neuroscience, 19(2), 341350. doi:10.1162/jocn.2007.19.2.341

Newport, R., \& Schenk, T. (2012). Prisms and neglect: What have we learned? Neuropsychologia, 50(6), 1080-1091. doi:10.1016/j. neuropsychologia.2012.01.023
Nicholls, M. E. R., Kamer, A., \& Loftus, A. (2008). Pseudoneglect for mental alphabet lines is affected by prismatic adaptation. Experimental Brain Research, 191(1), 109115. doi:10.1007/s00221-008-1502-x

Parton, A., Malhotra, P., \& Husain, M. (2004). Hemispatial neglect. Journal of Neurology, Neurosurgery, and Psychiatry, 75(1), 13-21.

Power, J. D., Mitra, A., Laumann, T. O., Snyder, A. Z., Schlaggar, B. L., $\&$ Petersen, S. E. (2014). Methods to detect, characterize, and remove motion artifact in resting state fMRI. NeuroImage, 84, 320 341. doi:10.1016/j.neuroimage.2013.08.048

Redding, G. M., Rossetti, Y., \& Wallace, B. (2005). Applications of prism adaptation: A tutorial in theory and method. Neuroscience and Biobehavioral Reviews, 29, 431-444.

Redding, G. M., \& Wallace, B. (1993). Adaptive coordination and alignment of eye and hand. Journal of Motor Behavior, 25(2), 75-88.

Redding, G. M., \& Wallace, B. (1997). Adaptive spatial alignment. Psychology Press.

Rorden, C., Karnath, H.-O., \& Bonilha, L. (2007). Improving lesionsymptom mapping. Journal of Cognitive Neuroscience, 19(7), 1081-1088. doi:10.1162/jocn.2007.19.7.1081

Rossetti, Y., Rode, G., Pisella, L., Farnè, A., Li, L., Boisson, D., \& Perenin, M. T. (1998). Prism adaptation to a rightward optical deviation rehabilitates left hemispatial neglect. Nature, 395(6698), 166169. doi: $10.1038 / 25988$

Rossit, S., McAdam, T., Mclean, D. A., Goodale, M. A., \& Culham, J. C. (2013). FMRI reveals a lower visual field preference for hand actions in human superior parieto-occipital cortex (SPOC) and precuneus. Cortex, 49(9), 2525-2541. doi:10.1016/j. cortex.2012.12.014

Roth, H. L., Lora, A. N., \& Heilman, K. M. (2002). Effects of monocular viewing and eye dominance on spatial attention. Brain, 125(Pt 9), 2023-2035.

Sarri, M., Greenwood, R., Kalra, L., Papps, B., Husain, M., \& Driver, J. (2008). Prism adaptation aftereffects in stroke patients with spatial neglect: Pathological effects on subjective straight ahead but not visual open-loop pointing. Neuropsychologia, 46(4), 1069-1080. doi:10.1016/j.neuropsychologia.2007.11.005

Serino, A., Angeli, V., Frassinetti, F., \& Làdavas, E. (2006). Mechanisms underlying neglect recovery after prism adaptation. Neuropsychologia, 44(7), 1068-1078. doi:10.1016/j. neuropsychologia.2005.10.024

Serino, A., Bonifazi, S., Pierfederici, L., \& Làdavas, E. (2007). Neglect treatment by prism adaptation: What recovers and for how long. Neuropsychological Rehabilitation, 17(6), 657-687. doi:10.1080 /09602010601052006

Singhal, A., Monaco, S., Kaufman, L. D., \& Culham, J. C. (2013). Human fMRI reveals that delayed action re-recruits visual perception. PloS One, 8(9), e73629. doi:10.1371/journal.pone.0073629

Smania, N., Fonte, C., Picelli, A., Gandolfi, M., \& Varalta, V. (2013). Effect of Eye Patching in Rehabilitation of Hemispatial Neglect. Frontiers in Human Neuroscience, 7(September), 527. doi:10.3389/fnhum.2013.00527

Smith, S. M., Jenkinson, M., Woolrich, M. W., Beckmann, C. F., Behrens, T. E. J., Johansen-Berg, H., ... Matthews, P. M. (2004). Advances in functional and structural MR image analysis and implementation as FSL. NeuroImage, 23(SUPPL. 1), 208-219. doi:10.1016/j.neuroimage.2004.07.051

Stratton, G. (1896). Some preliminary experiments on vision without inversion of the retinal image. Psychological Review, 3(6), 611617. doi: $10.1037 / \mathrm{h} 0072918$

Striemer, C. L., \& Danckert, J. A. (2010). Through a prism darkly: Reevaluating prisms and neglect. Trends in Cognitive Sciences, 14(7), 308-316. doi:10.1016/j.tics.2010.04.001

Striemer, C. L., Sablatnig, J., \& Danckert, J. A. (2006). Differential influences of prism adaptation on reflexive and voluntary covert 
attention. Journal of the International Neuropsychological Society, 12(3), 337-349.

Thompson, R. F., \& Spencer, W. A. (1966). Habituation: A model phenomenon for the study of neuronal substrates of behavior. Psychological Review, 73(1), 16-43. von Helmholtz, H. L. F. (1962). Helmholtz's treatise on Physiological Optics. (J. P. . Southall, Ed.). New York: New Dover (Original work published in 1866).

Walker, R., Young, A. W., \& Lincoln, N. B. (1996). Eye patching and the rehabilitation of visual neglect. Neuropsychological Rehabilitation, 6(3), 219-231. 